\title{
La subjectivité résiste !
}

\section{Personal Subjectivity Is still Resisting}

\author{
M.-F. Bacqué \\ (C) Lavoisier SAS 2021
}

Ce numéro 4 de l'année 2020 est riche en expériences qui restaurent la place de la subjectivité des personnes atteintes de cancer. Cela commence avec un peu de musique.

\section{La musique relâche les défenses, en conséquence penser à sa mort n'est plus interdit}

L'application Music Care ${ }^{1}$ a été étudiée en HAD avec une dizaine de patients. Le groupe témoin bénéficiait de son côté d'une séance de relaxation. Music Care est en général proposée aux personnes anxieuses, dépressives ou souffrant de douleurs aiguës ou chroniques. La plupart des patients ont pu échapper grâce à leur centration sur leur imaginaire, à l'état de maladie. En revanche, les différents morceaux leur ont aussi permis d'accéder puis de relâcher leurs pensées à la mort. Celles-ci ont été facilitées par l'isolement sensoriel dû au casque audio et à l'obturation de la lumière. Si certains se sont sentis « dans un cercueil », d'autres ont pu imaginer leur propre enterrement, mais de façon heureuse, comme si le déplacement habituel des pensées morbides ne fonctionnait plus. C'est ce qui fait conclure aux auteurs de cette recherche, Camille Valette de Poplavsky, Coline Windal, Camille Baussant-Crenn, Jérémy Martin et Carolina Baeza-Velasco, tous.tes psychologues et universitaires, que Music Care (qui, sur son site, présente plus de 150 études scientifiques) peut être vécue de façon ambiguë par des patients atteints de maladies mortelles. En libérant la psyché de leurs défenses, les patients envisageraient l'issue de leur vie, d'où la conclusion d'un accompagnement psychologique nécessaire afin de profiter de cette levée défensive ; et, si le patient le demande, de poursuivre les associations d'idées vers leurs thématiques existentielles.

M.-F. Bacqué ( $\square)$

SuLiSoM-UR3071, université de Strasbourg,

F-6700 Strasbourg, France

e-mail : bacque@unistra.fr

\footnotetext{
$\overline{{ }^{1} \text { www.music-care.com. }}$
}

\section{Comment croiser les représentations de la douleur et des souffrances entre les oncologues et les patients ?}

Toujours dans le registre de la place de la subjectivité dans l'expérience cancéreuse, Edith Salès-Wuillemin, Catherine Lejeune, Anthony Clain, Thomas Carrel et Adrien Dolard de l'université de Bourgogne-Franche-Comté et de l'équipe EPICAF et CIC Inserm 1432 ont analysé les systèmes de représentation de la douleur et de la souffrance en parallèle chez 20 patients et 13 médecins oncologues. Ils ont interprété les discours grâce au logiciel d'analyse lexicale Iramuteq ${ }^{\circledR}$. Ce qui frappe est que douleur et souffrance ne figurent pas dans le même champ sémantique. Par ailleurs, chaque groupe se représente la maladie au travers de l'autre : le médecin évoque la maladie du patient, le patient se réfère à la maladie traitée par le médecin et ressentie par lui. Si la douleur est associée par les patients au cancer, aux examens et aux effets secondaires des traitements, leur souffrance résulte de la peur de l'échec des traitements et des conséquences de la maladie en termes de détérioration physique, sociale et psychologique. Les médecins ont une vision beaucoup plus rationnelle de la douleur, qui reste LE symptôme de la maladie et le levier de la recherche thérapeutique. Le dialogue n'existe pas encore sur ces deux faces d'une plainte multiforme : (Quand) le patient parle de sa douleur, il cherche aussi à exprimer sa souffrance. Le médecin, pour sa part, retient la douleur et écarte l'expression de la souffrance car elle sort de son domaine de compétence. La souffrance n'est pas abordée par le médecin parce qu'elle renvoie à son impuissance.

\section{Un début de confinement difficile pour les patients atteints de cancer}

L'enquête BaroCov, sous la direction de Jean-Christophe Mino a recensé, à l'institut Curie, les préoccupations et les satisfactions de 2478 patients depuis le début de la Covid et jusqu'en mai 2020. En ces débuts de pandémie, les thèmes d'inconfort n'étaient pas tant le coronavirus que la maladie 
cancer et ses traitements. La perte de chance était citée explicitement : report de traitements, retard dans les consultations médicales, interventions repoussées. Cependant, l'autre face du confinement était aussi citée comme un bénéfice appréciable : temps libre, sentiment de paix, entourage restreint mais attentionné. Avec le recul d'une année, nous pouvons dire qu'il y a un an, la satisfaction du confinement ne s'était pas encore transformée en lassitude et en démotivation. De nombreux patients atteints de cancer ont vu leurs traitements remis en question et ont certainement été effrayés à l'idée de voir leur maladie passer derrière une épidémie aiguë que le risque de contamination poussait au premier plan. Certains en ont conçu une certaine culpabilité, car, nous l'entendons souvent, "il y a toujours un malade dans un état pire que le sien ». Cependant, les aléas de la propagation des variants prolongent la situation et rendent maintenant cruciales opérations et poursuites des traitements. Une telle enquête doit être reproduite une année après le premier confinement pour évaluer l'adaptation des patients mais aussi des services qui doivent maintenant compter avec des soignants épuisés.

\section{Une plainte subjective récurrente : celle du chemobrain}

Puisque nous nous penchons sur l'expression de la subjectivité dans ce numéro, voyons comment l'université de Caen, le centre François-Baclesse, la Ligue contre le cancer et l'association France Lymphome Espoir recherchent et interprètent la plainte cognitive des personnes traitées pour un lymphome. La Stratégie Cancer 2020-2030 annoncée par l'INCa propose la réduction des effets indésirables des traitements sur les fonctions cognitives. Le chemobrain, cette impression de " brouillard cognitif», a été précisé par Marie Lange, Florence Joly et leur équipe en 2019 en un Cancerrelated cognitive impairment. L'expérience subjective d'une altération cognitive est une des séquelles les plus remarquées et cependant tue la plupart du temps au regard du pronostic des lymphomes, surtout non hodgkiniens (qui présenteraient $55 \%$ de taux de survie à cinq ans). Les autoquestionnaires recueillis auprès de 643 personnes montrent, tous âges confondus, $62 \%$ de plaintes cognitives, particulièrement chez les femmes. Les plaintes, le malaise, les troubles vécus de façon globale et diffuse ne relèvent pas forcément uniquement des effets secondaires des traitements anticancéreux. La dépression, l'angoisse et la fatigue peuvent en effet majorer les difficultés de concentration, d'engrammage des informations et leur restitution. Les participants les plus jeunes et les femmes souhaitent trouver une réhabilitation cognitive qui pourrait éventuellement trouver une place dans une alliance des associations de soutien et des départements de soins de support. Mais pour une amélioration plus efficiente, les programmes devraient comprendre une approche plus globale. Si le chemobrain est vraisemblablement d'origine organique, il combine des éléments affectifs issus des représentations de la maladie cancer et de tous ses effets psychologiques. La rééducation n'est donc pas la seule option à mettre en place, elle relève d'une approche globale qui traite également l'expérience de la maladie.

\section{Le référentiel sur le deuil destiné aux soignants}

Jean-Sébastien Leplus-Habeneck, Voskan Kirakosyan, Léonor Fasse et Ophélie Soulié proposent une observation clinique d'un deuil pour illustrer l'intérêt du référentiel interrégional « Le Deuil ». L'AFSOS ${ }^{2}$, la SFAP ${ }^{3}$ et la SFFPO ${ }^{4}$ ont soutenu les travaux d'une cinquantaine de professionnels sous la direction d'Isabelle Lombard et d'Ophélie Soulié. Le référentiel est très complet, très loin de se tenir à une pure description symptomatique comme les grandes nosographies (DSM-5 et CIM-10 et 11). Le cas très intéressant de Sophie qui consulte 19 mois après la mort de son conjoint décrit de façon exemplaire son utilisation. " Hantée » par les images de la fin de Stéphane, elle n'a pas pu encore intégrer sa mort dans le sens de son histoire personnelle et familiale. La mort du proche est exclue de la continuité que chacun d'entre nous élabore inconsciemment avec le temps qui passe. Mais progressivement, elle va retrouver à travers la narration interne et externe que l'on construit, un moyen de s'accorder avec le reste de notre histoire. Tout cela n'est cependant qu'une représentation de notre vie et de la perte dans notre vie. Chez Sophie, les dernières images du mourant, les odeurs, les impressions psychiques sont prégnantes, les conflits internes ne sont pas élaborés. Ce qui signe le deuil compliqué est la croissance des difficultés à apaiser la révolte contre la mort et l'envahissement de toute la sphère psychosociale de Sophie : cauchemars, culpabilité, visions.

Le travail psychothérapique va permettre de comprendre que les difficultés de Sophie ne sont pas nouvelles. Elle a déjà vécu un deuil traumatique (le suicide inattendu de sa mère), sa famille, dysfonctionnelle, ne lui a pas donné la capacité de développer des liens affectifs sans ambivalence (habituelle certes mais dont la prise de conscience aurait dû conduire à une relation de réciprocité), enfin, elle a longtemps dénié la gravité de la maladie de son conjoint. Son refus de l'intervention d'une équipe mobile de soins palliatifs en prétextant le maintien d'une illusion chez elle et son mari, montre une idéalisation de sa capacité à résoudre les conflits dans son couple alors qu'ils étaient confrontés au départ de leur fils.

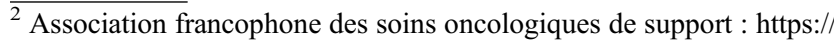
www.afsos.org.

${ }^{3}$ Société française d'accompagnement et de soins palliatifs.

${ }^{4}$ Société française et francophone de psycho-oncologie.
} 


\section{D'autres subjectivités que la mienne...}

Paraphraser Emmanuel Carrère est sans doute un peu facile ; cependant, les enseignements de ce numéro de PsychoOncologie relèvent de ce questionnement. Autant de patients, autant de subjectivités. Mais attention, la conclusion est la même pour les soignants, malgré toute la neutralité dont ils souhaiteraient imprégner leur discours et leurs actes...

La recherche de Rachel Ferrere et de Jacqueline Wendland compare des jeunes femmes présentant un cancer périnatal à des femmes avec un cancer du sein d'âge comparable (l'âge moyen est de 36,7 contre 38,6 ans). Les jeunes mères ont montré beaucoup plus de difficultés que le groupe témoin. Leurs mécanismes de défense étaient souvent massifs : déni de la réalité, évitement de l'angoisse, centration sur les difficultés d'adaptation au bébé. Le contraste entre cancer et maternité était pourvoyeur de clivage et de réactions abruptes plutôt que d'adaptation. Plus que jamais l'accompagnement psychologique de ces patientes semble le moyen de faciliter leur retour vers leur vie et celle de leur famille débutante ${ }^{5}$.

L'étude de nos collègues tunisiens, Asma Ghorbel et al. sur l'image du corps de 100 femmes suivies par leur service de radiothérapie pour un cancer de l'endomètre ou du col utérin montre combien la culture influe sur l'expérience propre de son corps. L'absence de communication avec le conjoint comme avec l'équipe soignante contribue largement à l'absence du soutien familial, l'altération de la relation de couple et de la relation sexuelle et la mauvaise tolérance de la chirurgie et la curiethérapie utérovaginale. L'âge des femmes, en revanche, semble réduire avec son avancée, les troubles de l'image corporelle. Beaucoup a été dit sur les représentations de ces cancers qui touchent les organes sexuels. Cependant, la Tunisie pas plus que le Maroc ne présentent de taux plus importants de troubles de l'image du corps par rapport à ceux des pays occidentaux. Le fantasme de contagion du cancer par le truchement de la sexualité est sans doute plus explicite. Quant au lien entre niveau socioéconomique et moindre intérêt pour l'esthétique physique des femmes, il semble que ce résultat soit composite, à relier avec les stéréotypes sociaux ou les préjugés nourris par nos différences culturelles. Remercions nos collègues du département de radiothérapie de l'institut Salah-Azaïz de Tunis pour cette étude très détaillée et exemplaire des recherches mixtes joignant les données socio-économiques aux particularités médicales et psychologiques de patient.es atteint.es de cancer.

Les avancées de la psychologie et de la médecine montrent combien celles-ci ne peuvent qu'être de plus en plus personnalisées. Cependant, les sciences de la subjectivité sont encore embryonnaires, car comment allier cas singulier avec résultats pour le plus grand nombre ? Les méthodes mixtes donnent une partie de la réponse, mais il reste que la lisibilité des résultats est complexe et parfois nous submerge, car notre attention est limitée. Cependant, les différentes études présentées dans ce numéro 4 de PsychoOncologie ont valorisé les expériences subjectives des patients comme des soignants en nous donnant une vision diversifiée de notre discipline. Puisse celle-ci nous amener à la subtilité et la nuance pour aider nos patients.

\footnotetext{
$\overline{5}$ Rappelons le beau numéro de Psycho-Oncologie, $\mathrm{n}^{\mathrm{o}}$ 2, 2009 sur Cancer et grossesse.
} 